\title{
CONOCIENDO EL UNIVERSO, UN poco más. Premio Nobel de FísICA 2019
}

\author{
Knowing the Universe, a little more. 2019 Nobel Prize in Physics
}

Juvenal Castromonte Salinas ${ }^{1}$

\section{RESUMEN}

El Premio Nobel de Física 2019 fue ganado por tres investigadores astrofísicos. Este artículo resume la contribución de J. Peebles sobre sus predicciones teóricas en cosmología, y de M. Mayor y D. Queloz por el descubrimiento de los exoplanetas.

Palabras claves: Premio Nobel, física, cosmología, astrofísica, exoplaneta.

\begin{abstract}
The 2019 Nobel Prize in Physics was awarded to three astrophysics researchers. This review summarizes the contribution of J. Peebles about his theoretical predictions on cosmology and M. Mayor and D. Queloz about the discovery of exoplanets.
\end{abstract}

Keywords: Nobel Prize, physics, cosmology, astrophysics, exoplanet.

La cúspide de la carrera artística para un actor es ganar el Oscar, para un deportista de elite es ganar el oro olímpico y, en el mundo científico, el mayor galardón es el Premio Nobel. Este año, en el área de la física, los ganadores del premio fueron J. Peebles, M. Mayor y D. Queloz.

El premio se dividió en dos partes, y de alguna manera representa una quimera, en el sentido de que tiene la cabeza de un animal y cuerpo

1 Físico, profesor principal. Sección de Física, Informática y Matemáticas, Facultad de Ciencias y Filosofía, Universidad Peruana Cayetano Heredia.

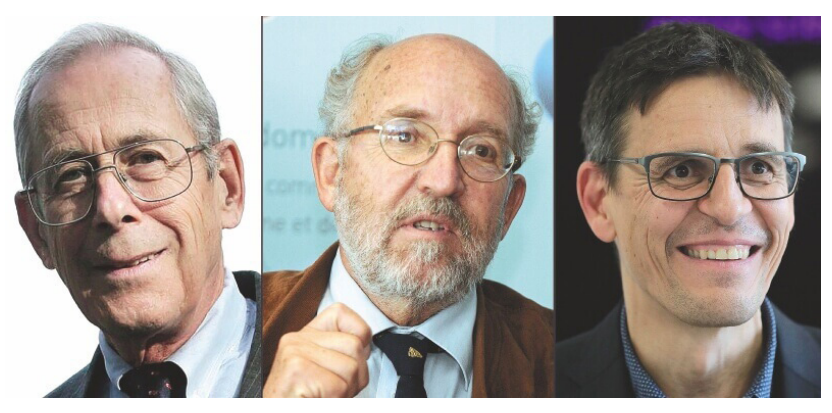

De izquierda a derecha, los astrofísicos James Peebles (EE.UU.), Michel Mayor (Suiza) y Didier Queloz (Suiza).

de otro. Una parte corresponde a la cosmología y la otra a la búsqueda de exoplanetas, dos áreas diferentes del conocimiento. Aunque, de manera condicional, se encuentran dentro de un tema común: el estudio del Universo. La primera parte del premio se le concedió a J. Peebles por sus descubrimientos (predicciones) teóricos en cosmología; la segunda parte se dividió entre M. Mayor y D. Queloz por el primer descubrimiento de un exoplaneta. Sin embargo, se debe tener presente que los galardonados tuvieron que esperar bastante tiempo por el premio. Los trabajos claves de Peebles fueron realizados en la segunda mitad de los años 1960, mientras que Mayor y Queloz descubrieron "su" exoplaneta en 1995. 


\section{Comprendiendo al Universo}

Los trabajos de Peebles sobre cosmología teórica tuvieron una fuerte repercusión para el desarrollo de la astrofísica a su estado actual. Entre sus trabajos más importantes que se incluyen en el Nobel de este año se tiene:

- Estudio sobre la radiación residual (1965), en Princeton, bajo la dirección de Dicke construyeron un telescopio para medir la radiación residual, basados en el antíguo modelo del Universo caliente de Gamov, entonces ya casi olvidado.

Durante la construcción del telescopio, en otro laboratorio de los EE.UU., bajo la dirección de Burke, sus colaboradores Penzias y Wilson hacían pruebas con un telescopio y se toparon con un ruido residual, de 3 grados de temperatura que no podían excluir. Burke, conocía las investigaciones del grupo de Princeton, y decidió comunicar a Dicke su hallazgo, quien al colgar el teléfono dijo "Boys, we were scooped", sintiendo que habían sido anticipados con los resultados obtenidos. Así, en 1965, el Astrophysical Journal publicó dos artículos, el de Wilson y Penzias sobre el descubrimiento del ruido y el de Peebles y Dicke acerca de los aspectos teóricos de este mismo fenómeno.

En 1978 Penzias y Wilson fueron galardonados con el Premio Nobel por el descubrimiento dela radiación residual. Peebles y Dicke se quedaron a un paso del Nobel. Debe hacerse notar que Peebles no solo predijo la existencia de la radiación residual sino también describió sus propiedades. Además, mostró la importancia de la radiación residual en la formación de las galaxias, adicionando al modelo del Universo la energía oscura de densidad negativa.
- Estudio de la núcleo-síntesis primordial en el Universo caliente (1966). La formación de elementos no pesados debido a las secuelas del Big bang.

- Problemas fundamentales sobre la expansión del Universo en etapas tempranas y en la época actual.

Efectivamente, en los años 1960, estos temas eran estudiados por muchos grupos. Todos ellos obtuvieron resultados importantes pero dar una descripción completa y sintetizar las diferentes investigaciones en cosmología y explicarlo de la manera mas simple posible solo pudo ser hecho por Peebles y su grupo, interpretando la radiación y descubriendo nuevos procesos físicos.

Los fundamentos teóricos establecidos por Peebles, actualmente, forman parte de los conceptos básicos del entendimiento de la historia de nuestro universo, desde la gran explosión (Big bang) hasta nuestros días. Alrededor de 400 mil años después de la gran explosión, el Universo, aun turbio, se transformó en transparente para la luz. Esta radiación residual es la que actualmente nos permite conocer algunos secretos del Universo, como por ejemplo cuándo se formaron las primeras estrellas y galaxias.

Estas predicciones fueron verificadas por los satélites COBE, WMAP y Planck. Los cálculos realizados por los cosmólogos, complementados y corregidos con las mediciones de los satélites, ahora nos permiten decir que el Universo está formado por el $5 \%$ de materia que percibimos normalmente, $26 \%$ de materia oscura y $69 \%$ de energía oscura. 


\section{EN LO MÁS LEJANO DEL UNIVERSO}

La segunda mitad del Premio Nobel de Física del 2019 se concedió a Queloz y a su discípulo M. Mayor por el descubrimiento, realizado, en octubre de 1995, de un exoplaneta y 51 estrellas de la constelación Pegaso. Las investigaciones de estos científicos, de inmediato, generaron una oleada de descubrimientos similares. A la fecha, ya se conocen más de 4000 exoplanetas en la Vía Láctea y su número sigue creciendo. Algunos de ellos se encuentran en la zona de habitabilidad, es decir, hipotéticamente en ellos podría existir vida. Figura 1

Lo que se encontró fueron los desplazamientos periódicos de las líneas espectrales de la estrella P51, que es similar al sol. Los desplazamientos de las líneas se producen por el efecto Doppler - la superficie de la estrella o se acerca, o se aleja de "nosotros". La interpretación natural que se dio es que la estrella gira alrededor del centro de gravedad común con cierto planeta. Este método se denomino método de la velocidad radial. Figura 2.

De la magnitud del desplazamiento Doppler y su periodo se concluye que el planeta tiene una masa similar a la de Júpiter: que gira alrededor de su estrella en 4 días y está 20 veces mas cerca a su estrella que la Tierra al Sol. De acuerdo a la teoría vigente, bajo esas condiciones es imposible la existencia de un planeta, menos aun una gigante, ya que al estar tan cerca a su estrella las fuerzas centrípetas que allí se tienen son demasiado intensas que no permitirían el crecimiento del planeta. Por esta razón, en un inicio, la comunidad científica asumía que no se trataba de un planeta, sino que era la "respiración" (fluctuación) periódica de una estrella. Efectivamente, la "respiración" de las estrellas puede producir una variación sinusoidal de la velocidad de sus rayos de luz; $\mathrm{y}$, también se debe tener en cuenta que cambia su luminosidad, pero de manera diferente a la de los planetas -con otra amplitud de fase. Sin embargo, para el Pegaso 51, la variación de su luminosidad sucedia exactamente igual a la de cualquier planeta. Finalmente, luego de algunos meses, la comunidad científica reconoció el descubrimeinto de los exoplanetas. Figura 3.

La luminosidad de los planetas es el reflejo de la luz, por lo que, incluso a distancias cercanas en términos astronómicos, no es

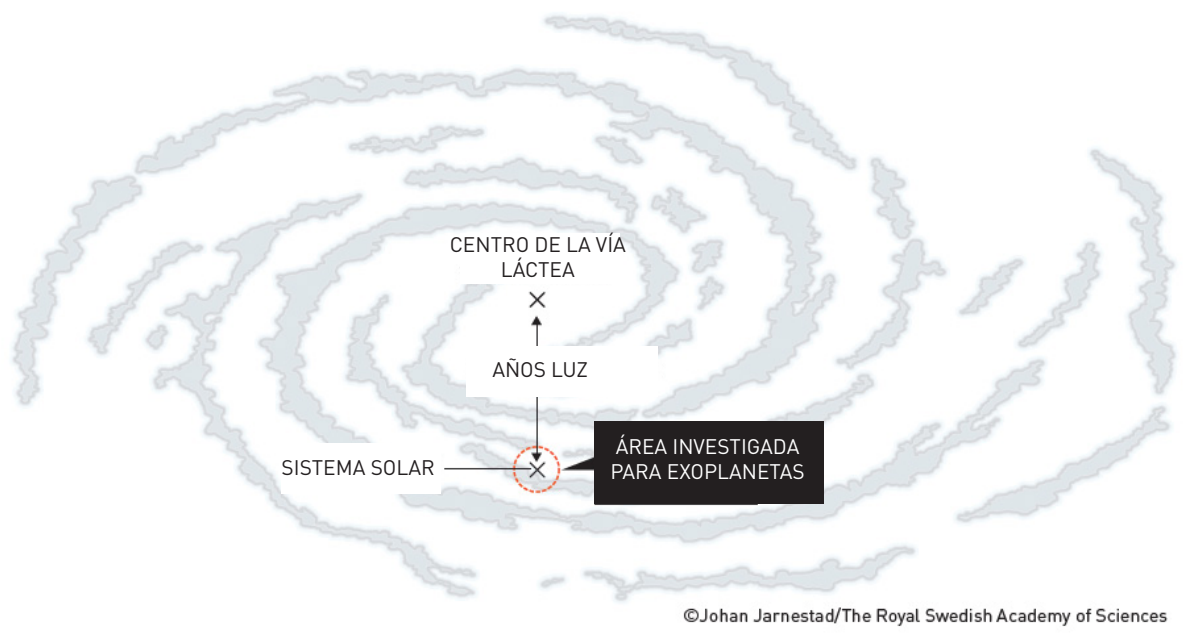

Figura 1. El sistema solar en la Vía Láctea. 


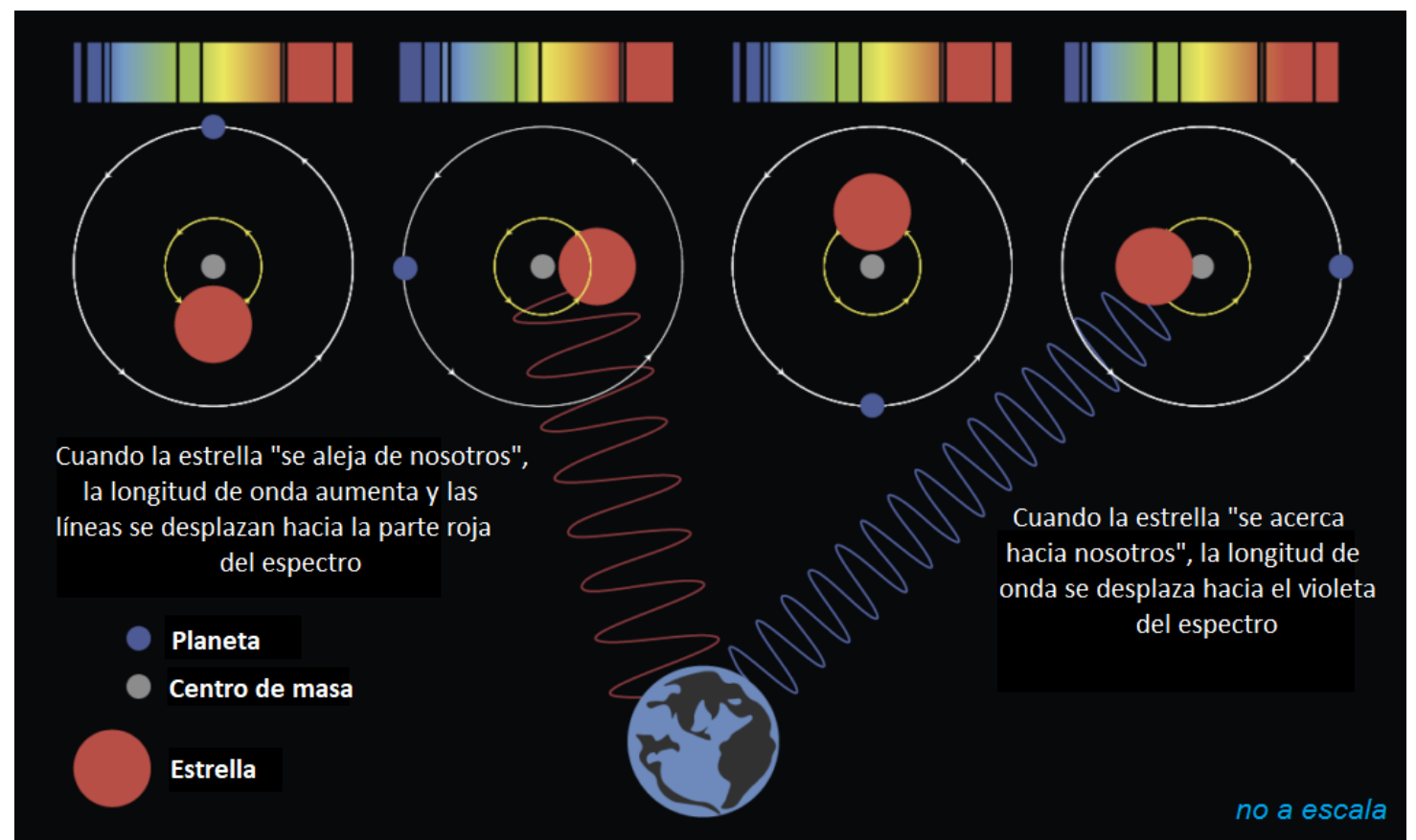

Figura 2. Efecto Doppler del exoplaneta P51.
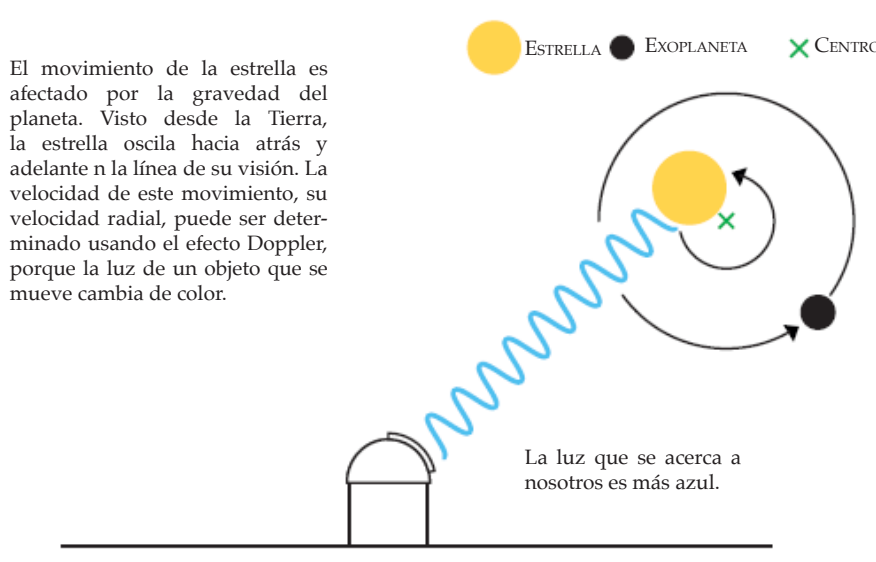

Velocidad DE LA ESTRELLA

HACIA LA TIERRA $(\mathrm{M} / \mathrm{s})$

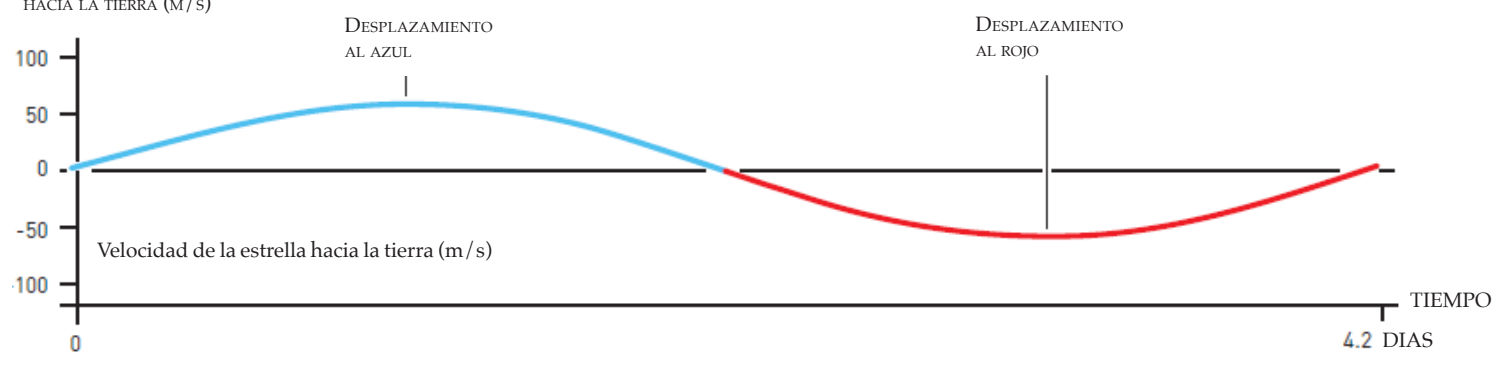

Q Johan Jarnestad/The Royal Swedish Academy of Sciences

Figura 3. Método de la velocidad radial para la detección de exoplanetas. 
simple poder verlos. Es el caso de Neptuno, que fue descubierto de manera indirecta, por su influencia en la órbita de Urano. Esto hace que la dificultad de encontrar un planeta fuera del sistema solar sea mucha mayor. El método usado por Queloz y Mayor fue el método indirecto de la velocidad radial.

El método consiste en entender el hecho de que el planeta y su estrella giran alrededor del centro de masa común a ambas. Así, resulta que el planeta se acerca o se aleja de la Tierra, por lo que de acuerdo con el efecto Dopler, la luz del planeta se va tornando azul (acercándose al violeta) cuando el planeta se acerca y se hace roja cuando se aleja.

Sin embargo, como ya se mencionó anteriormente, observar este efecto es bastante complicado. La masa de la estrella es mucho mayor que la masa del planeta, lo que exige contar con instrumentos de precisión para las mediciones. Por ejemplo, Jupiter obliga moverse al Sol a una velocidad de $12 \mathrm{~m} / \mathrm{s}$, mientras que la Tierra apenas a $10 \mathrm{~cm} / \mathrm{s}$. Mayor y sus colegas construyeron el espectrógrafo ELOIDE para el Observatorio Haute-Provence de Ginebra, cuya precisión era 20 veces mayor que el espectrógrafo anterior que se usaba. Esto permitio fijar las oscilaciones de estrellas con velocidades de $10 \mathrm{~m} / \mathrm{s}$, por lo que ahora resulta entendible el por qué primero se descubieran planetas que tenian parámetros similares a los de Jupiter antes que a los de la Tierra. Lo más interesante es que el planeta descubierto estaba 20 veces más cerca a su estrella que la Tierra al Sol. Hasta entonces se consideraba que los demás sistemas planetarios estaban formados de manera similar al solar: los planetas pequeños en las órbitas interiores, luego iban los planetas gaseosos gigantes $\mathrm{y}$ finalmente los gélidos. Ahora, gracias a estos descubrimientos, sabemos que no todos los sistemas planetarios son similares.

Mayor y Queloz fueron afortunados al ser los primeros en descubrir un exoplaneta, pues la busqueda la realizaban varios grupos de científicos. Algunas semanas después, del descubrimiento del P51, se tenian comunicaciones sobre descubrimientos de otros dos planetas del tipo de "Júpiter caliente". Actualmente, el número de exoplanetas se acerca al millar. El descubrimiento de Mayor y Queloz hizo que la busqueda de exoplanetas se considere una de las áreas de moda en la astrofísica. Inicialmente se descubrieron planetas grandes, del tipo Júpiter, posteriormente se aprendio a encontrar planetas de menor tamaño, mas similares a la Tierra.

Ahora, la busqueda es de planetas "habitados", incluso si solo son habitados por virus o bacterias. Indudablemente, quienes abrieron el camino en esta busqueda tienen merecido el Premio Nobel.

\section{Referencias Bibliográficas}

1. Press release: The Nobel Prize in Physics. 2019

2. Butler RP, Marcy GW, Williams E, et al. Attaining Doppler Precision of $3 \mathrm{M} \mathrm{s}-1$. Publications of the Astronomical Society of the Pacific. 1996;108:500.

3. Mayor M, Queloz D. A Jupiter-mass companion to a solar-type star. Nature. 1995;378:355-359.

\section{CoRrespondencia:}

juvenal.castromonte@upch.pe

FECHA DE RECEPCIÓN: 22-01-2020.

FECHA DE ACEPTACIÓN: 08-02-2020. 